\title{
Atherogenic Index of Plasma Is a Potential Biomarker for Severe Acute Pancreatitis: A Prospective Observational Study
}

\author{
Seung Kook Cho ${ }^{1}$, Jae Woo Kim ${ }^{2}$, Ji Hye Huh ${ }^{3, *}$ and Kyong Joo Lee ${ }^{2, *}$ (i) \\ 1 Department of Internal Medicine, Gangwondo Wonju Medical Center, Wonju 26448, Korea; \\ lukechosk@gmail.com \\ 2 Department of Internal Medicine, Yonsei University Wonju College of Medicine, Wonju 26426, Korea; \\ jawkim96@yonsei.ac.kr \\ 3 Division of Endocrinology and Metabolism, Department of Internal Medicine, Hallym University Sacred \\ Heart Hospital, Anyang 14068, Korea \\ * Correspondence: png1212@hanmail.net (J.H.H.); smild123@yonsei.ac.kr (K.J.L.)
}

Received: 23 August 2020; Accepted: 14 September 2020; Published: 15 September 2020

\begin{abstract}
Background: The atherogenic index of plasma (AIP) reflects the levels of triglycerides (TG) and high-density lipoprotein (HDL) cholesterol. The purpose of this study was to assess the relationship between the AIP and severe acute pancreatitis (SAP). Materials and methods: Patients with acute pancreatitis (AP) were prospectively enrolled from March 2015 to June 2019. The severity of AP was classified according to the 2012 revised Atlanta classification. Mild and moderately severe AP were categorized as non-SAP. The AIP is calculated as $\log (\mathrm{TG} / \mathrm{HDL})$. Results: A total of 323 patients were enrolled. The etiologies of AP were gallstone in 171 patients $(52.9 \%)$, alcohol in 122 patients $(37.8 \%)$, and hypertriglyceridemia in 30 patients $(9.3 \%)$. Twenty-four patients $(7.4 \%)$ were classified as SAP. The AIP was significantly higher in the SAP group compared to the non-SAP group $(p<0.001)$. The AIP was positively correlated with the Atlanta classification $(R=0.256$, $p<0.001)$. In multivariate analysis, the AIP was found to be an independent predictive factor for SAP $(\mathrm{OR}=4.571 ; \mathrm{CI}=1.913-10.922 ; p=0.001)$. Conclusions: The AIP is a potential biomarker for the prediction of SAP in clinical practice. This result provides that impaired lipid metabolism is associated with the severity of pancreatitis.
\end{abstract}

Keywords: acute pancreatitis; atherogenic index of plasma; biomarker; severity; Atlanta classification

\section{Introduction}

Acute pancreatitis (AP) is an inflammatory disease of the pancreas which can cause local injury, systemic inflammatory response syndrome (SIRS), and organ failure (OF). The incidence of AP is increasing, with an annual incidence of 5-30 per 100,000 individuals [1,2]. The overall mortality rate for $\mathrm{AP}$ is approximately $5 \%$, and is up to $30 \%$ in patients who experience SIRS and OF [3]. According to the 2012 revised Atlanta classification, severe acute pancreatitis (SAP) is defined when there is persistent OF ( $>48 \mathrm{~h}$ ) [4]. The predicted mortality of SAP is approximately $10 \%$, as compared with less than $1 \%$ in patients whose AP is predicted to be mild [5]. Therefore, the prediction of the severity of AP is crucial to triage patients during the initial diagnosis. Early intensive care is necessary for patients with SAP in order to reduce the morbidity and mortality. Complex scoring systems have been developed to determine the severity of AP, including the Ranson scoring system, Acute Physiology and Chronic Health Evaluation (APACHE) II, computed tomography scoring index (CTSI), and Bedside Index for the Severity in Acute Pancreatitis (BISAP) [6-9]. Inflammatory markers such as C-reactive protein $(\mathrm{CRP})$ or procalcitonin are also used as single parameters for the prediction of the severity of AP [10]. 
The atherogenic index of plasma (AIP) reflects the levels of triglycerides (TG) and high-density lipoprotein (HDL) cholesterol, and is calculated as $\log (\mathrm{TG} / \mathrm{HDL})$. The AIP has been used to quantify blood lipid levels and as a robust biomarker of dyslipidemia and atherogenicity. It is also considered an indicator of metabolic syndrome and coronary syndrome [11,12]. In this regard, local and systemic lipotoxicity has been recognized as an important risk factor for SAP or multisystem OF in AP [13]. Previous studies have reported that low HDL cholesterol is an independent predictor for SAP and longer hospitalization for patients with AP $[14,15]$. Elevated serum TG is also a well-known risk factor of persistent $\mathrm{OF}$ in AP [16]. Considering the close relationship between impaired lipid metabolism and SAP, we assume that the AIP may be a predictor of the severity of AP.

However, the relationship between the AIP and the severity of AP remains unknown. Therefore, we determined the relationship between the AIP and the severity of AP and evaluated the predictive ability of the AIP for the severity of AP compared with other scoring systems.

\section{Materials and Methods}

\subsection{Study Population}

Patients diagnosed with AP at Yonsei University Wonju College of Medicine between March 2015 and June 2019 were enrolled in this prospective observational study. The study protocol was approved by the Internal Review Board for Human Research of Yonsei University Wonju College of Medicine (approval no. CR315005-002). This study was performed in accordance with the relevant guidelines and regulations. Written informed consent was obtained from all patients.

\subsection{Diagnostic Criteria}

The diagnosis of AP was based on the presence of two of the following three criteria: (1) upper abdominal pain, (2) serum amylase and/or lipase $\geq 3$ times the upper normal limit, and (3) characteristic imaging on a radiological study [17]. The etiology of AP included gallstones, alcohol consumption, and high TG. The exclusion criteria were the following: (1) age < 18 years old, (2) idiopathic pancreatitis, (3) patients with incomplete lipid profile data, (4) chronic pancreatitis, and (5) recurrent pancreatitis.

\subsection{Data Collection}

Clinical data and blood samples were obtained upon the patients' diagnoses of AP. The presence of hypertension, diabetes mellitus, alcohol consumption, and smoking was recorded. The patients' body mass index (BMI) was calculated using their height and weight. Hemoglobin, hematocrit, white blood cell (WBC) count, blood urea nitrogen, creatinine, lactate dehydrogenase, aspartate aminotransferase, $\mathrm{CRP}$, procalcitonin, amylase, and lipase were recorded at the time of admission. Abdominal computed tomography (CT) scans were performed to diagnose AP and differentiate AP from other diseases. Once AP was diagnosed, the TG and HDL levels were measured as soon as possible. Additionally, scoring systems such as the Ranson score, BISAP, and CTSI were calculated. The AIP was defined as $\log$ (TG/HDL). The severity of AP was evaluated according to the 2012 revised Atlanta classification and recorded as mild, moderately severe, or severe AP [4]. Mild AP is defined by the absence of OF and local or systemic complications. Moderately severe AP is described as transient OF that resolves within $48 \mathrm{~h}$. SAP is described as persistent OF. Non-SAP included mild AP and moderately severe AP.

\subsection{Statistical Analysis}

Categorical variables were presented as frequencies and percentages. Continuous variables were presented as means ( \pm standard deviation) or medians with ranges. The Pearson rank method was used to evaluate the correlation between the AIP and the Atlanta classification. After adjustments for confounding factors, the odds ratios for SAP were evaluated using univariate and multivariate logistic regression analyses in order to determine the predictive factors of SAP among all the patients with AP and the predictive factors for intensive care unit (ICU) admission. The accuracy of the prediction power 
for SAP was determined by the area under the curve (AUC) using receiver operating characteristic (ROC) curves. AUC values were used to compare the predictive abilities of SAP using the AIP and scoring systems. A $p$ value $<0.05$ was considered statistically significant. Analyses were performed using SPSS version 23 (IBM, Armonk, NY, USA).

\section{Results}

\subsection{Baseline Characteristics of Patients}

A total of 323 patients were included in this study (Figure 1). Table 1 describes the characteristics of the participants. The mean age was $56.2 \pm 18.3$ years, and $271(68.8 \%)$ patients were male. The etiologies of AP were gallstones in 171 patients (52.9\%), alcohol over-consumption in 122 patients $(37.8 \%)$, and hypertriglyceridemia in 30 patients (9.3\%). According to the 2012 revised Atlanta classification, the severity of AP was mild in 181 patients (56\%), moderately severe in 118 patients (36.5\%), and severe in 24 patients (7.4\%). The Ranson score, CTSI, and BISAP were higher in the SAP group compared to the non-SAP group $(p<0.001)$. The number of ICU admissions, mortality, and the duration of hospital stays were significantly higher in the SAP group compared to the non-SAP group $(p<0.001)$. The levels of CRP, procalcitonin, HDL, and TG were not different between the two groups, but the level of the AIP was significantly higher in the SAP group ( $1.0 \pm 0.6$ vs. $0.5 \pm 0.5, p<0.001)$.

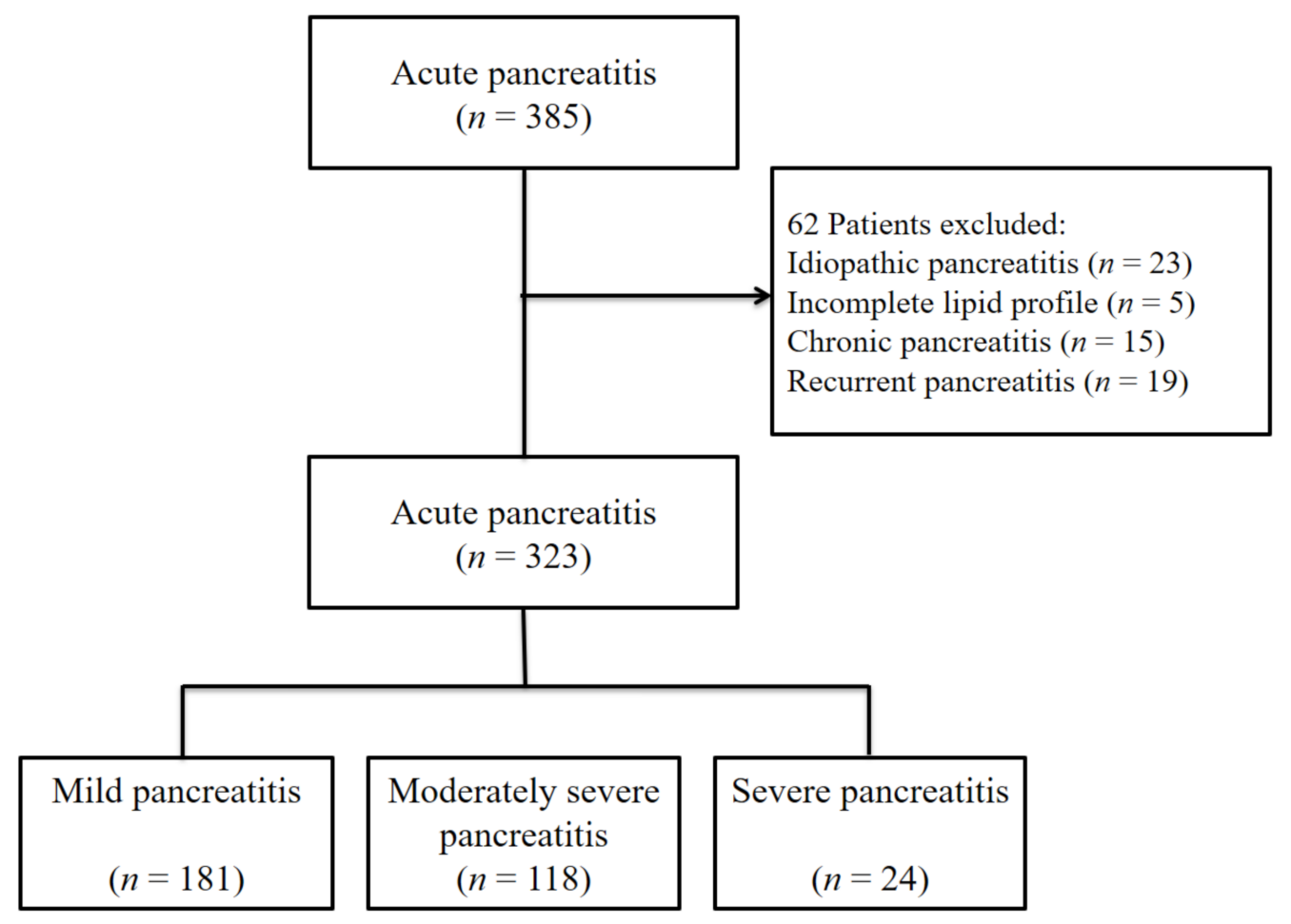

Figure 1. Flow chart of the study population. 
Table 1. Baseline characteristics of all the patients.

\begin{tabular}{|c|c|c|c|c|}
\hline Variable & $N=323$ & $\begin{array}{c}\text { SAP } \\
(n=24)\end{array}$ & $\begin{array}{l}\text { Non-SAP } \\
(n=299)\end{array}$ & $p$-Value \\
\hline Gender (Male: Female) & $271(68.8 \%): 123(31.2 \%)$ & 14(58.3\%):10(41.7\%) & 209(69.9\%):90(30.1\%) & 0.238 \\
\hline Age, years & $56.2 \pm 18.3$ & $54.7 \pm 21.4$ & $56.3 \pm 18.1$ & 0.689 \\
\hline Etiology of acute pancreatitis & & & & 0.134 \\
\hline Gallstone & $171(52.9 \%)$ & $8(33.3 \%)$ & $163(54.5 \%)$ & \\
\hline Alcohol & $122(37.8 \%)$ & $13(54.2 \%)$ & $109(36.5 \%)$ & \\
\hline Hypertriglyceridemia & $30(9.3 \%)$ & $3(12.5 \%)$ & $27(9 \%)$ & \\
\hline Smoking & $143(44.3 \%)$ & $12(50 \%)$ & $131(43.8 \%)$ & 0.557 \\
\hline Hypertension & $124(38.4 \%)$ & $10(41.7 \%)$ & $114(38.1 \%)$ & 0.732 \\
\hline Diabetes Mellitus & $87(26.9 \%)$ & $6(25 \%)$ & $81(27.1 \%)$ & 0.824 \\
\hline Body mass index, $\mathrm{kg} / \mathrm{m}^{2}$ & $24.3 \pm 4.6$ & $25.6 \pm 5.4$ & $24.2 \pm 4.5$ & 0.162 \\
\hline \multicolumn{5}{|l|}{ Atlanta classification } \\
\hline Mild & $181(56 \%)$ & & & \\
\hline Moderately severe & $118(36.5 \%)$ & & & \\
\hline Severe & $24(7.4 \%)$ & & & \\
\hline Ranson & $2.7 \pm 1.6$ & $4.2 \pm 1.3$ & $2.6 \pm 1.6$ & $<0.001$ \\
\hline CTSI & $2.1 \pm 1.4$ & $3.5 \pm 1.4$ & $1.9 \pm 1.3$ & $<0.001$ \\
\hline BISAP & $1.3 \pm 1.1$ & $2.7 \pm 1.2$ & $1.2 \pm 1.0$ & $<0.001$ \\
\hline Hospital stay, days & $6.5 \pm 5.5$ & $13.2 \pm 7.1$ & $6.0 \pm 5.0$ & $<0.001$ \\
\hline Intensive care unit admission & $40(12.4 \%)$ & $14(58.3 \%)$ & $26(8.7 \%)$ & $<0.001$ \\
\hline Mortality & $6(1.9 \%)$ & $5(20.8 \%)$ & $1(0.3 \%)$ & $<0.001$ \\
\hline \multicolumn{5}{|l|}{ Laboratory findings } \\
\hline C-reactive protein, $\mathrm{mg} / \mathrm{dL}$ & $4.6 \pm 7.1$ & $6.4 \pm 9.6$ & $4.5 \pm 6.8$ & 0.355 \\
\hline Procalcitonin, ng/mL & $5.4 \pm 21.8$ & $19.8 \pm 52.6$ & $4.3 \pm 16.8$ & 0.163 \\
\hline $\mathrm{HDL}, \mathrm{mg} / \mathrm{dL}$ & $37.7 \pm 18.3$ & $33.9 \pm 18.9$ & $38.0 \pm 18.3$ & 0.296 \\
\hline Triglycerides, mg/dL & $302.1 \pm 723$ & $544.3 \pm 860.5$ & $282.7 \pm 709.2$ & 0.088 \\
\hline AIP & $0.6 \pm 0.5$ & $1.0 \pm 0.6$ & $0.5 \pm 0.5$ & $<0.001$ \\
\hline
\end{tabular}

Results are presented as the mean \pm standard deviation or median; CTSI: computed tomography severity index; BISAP: the Bedside Index for Severity in Acute Pancreatitis; HDL: high-density lipoprotein; AIP: atherogenic index of plasma.

\subsection{The AIP and Scoring Systems for Predicting SAP}

We calculated the AUCs of various scoring systems and the AIP for predicting SAP (Table 2). The AUC of BISAP showed the greatest accuracy for the prediction of SAP (AUC $=0.813$ ). The AUC of the AIP for prediction of SAP was 0.709, which was not significantly different from the AUC of other scoring systems (Figure 2). The AIP was positively correlated with WBC $(R=0.146 ; p=0.009)$, $\operatorname{CRP}(R=0.236 ; p<0.001)$, and BMI $(R=0.134 ; p=0.016)$ (Table 3$)$. Furthermore, the AIP was positively correlated with the Atlanta classification $(R=0.256, p<0.001)$.

Table 2. Area under the curve for predicting severe acute pancreatitis.

\begin{tabular}{ccccc}
\hline Variable & AUC & Standard Error & $\mathbf{9 5 \%}$ CI & $p$-Value \\
\hline AIP & 0.709 & 0.056 & $0.600-0.819$ & 0.001 \\
TG & 0.683 & 0.040 & $0.606-0.761$ & 0.003 \\
HDL & 0.371 & 0.057 & $0.260-0.482$ & 0.035 \\
CTSI & 0.805 & 0.037 & $0.734-0.877$ & $<0.001$ \\
Ranson score & 0.778 & 0.038 & $0.704-0.852$ & $<0.001$ \\
BISAP & 0.813 & 0.040 & $0.734-0.892$ & $<0.001$
\end{tabular}

AUC: area under the curve; CI: confidence of interval; AIP: atherogenic index of plasma; TG: triglycerides; HDL: high-density lipoprotein; CTSI: computed tomography severity index; BISAP: the Bedside Index for Severity in Acute Pancreatitis. 


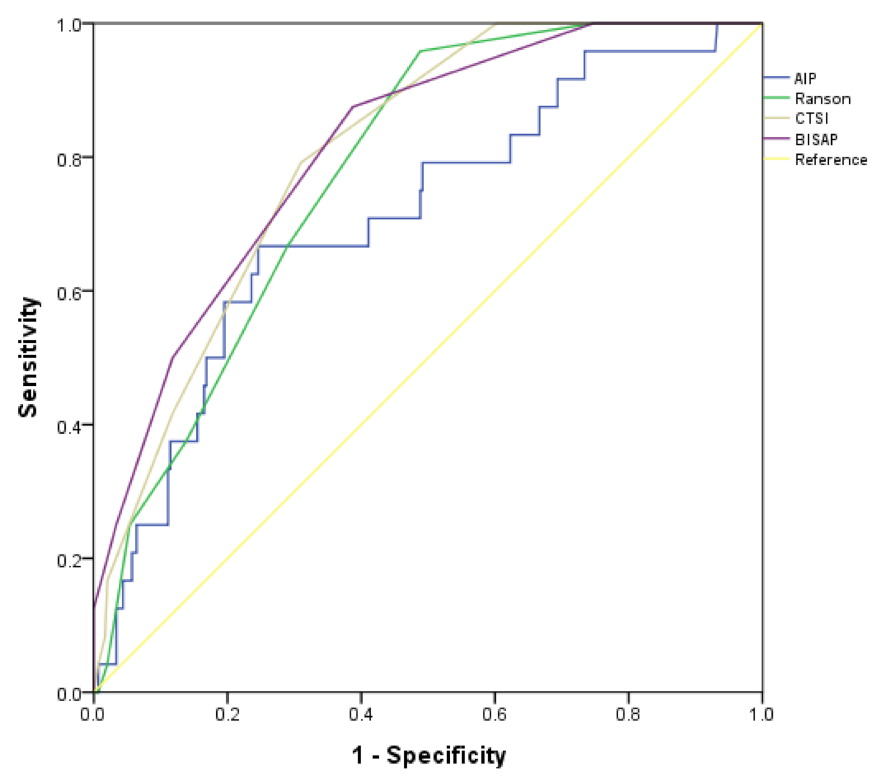

Figure 2. Receiver operator characteristic curve of various factors as predictors of severe acute pancreatitis. AIP: atherogenic index of plasma; CTSI: computed tomography scoring index; BISAP: the Bedside Index for Severity in Acute Pancreatitis.

Table 3. The correlation of the AIP and other variables.

\begin{tabular}{ccccc}
\hline Statistics & Body Mass Index & White Blood Cell & C-Reactive Protein & Atlanta Classification \\
\hline AIP & & & & \\
$R$ & 0.134 & 0.146 & 0.236 & 0.256 \\
$p$-Value & 0.016 & 0.009 & $<0.001$ & $<0.001$ \\
\hline
\end{tabular}

Pearson rank method was used. AIP: atherogenic index of plasma.

\subsection{Predictive Factors Affecting SAP and ICU Admission}

In the univariate analysis, the procalcitonin $(\mathrm{OR}=1.015 ; p=0.008)$ and $\mathrm{AIP}(\mathrm{OR}=3.119 ; p<0.001)$ were related to SAP. In the multivariate analysis, the AIP (OR $=4.571 ; \mathrm{CI}=1.913-10.922 ; p=0.001)$, alcohol $(\mathrm{OR}=5.782 ; \mathrm{CI}=1.111-30.088 ; p=0.037)$, and procalcitonin $(\mathrm{OR}=1.013 ; \mathrm{CI}=1.000-1.025$; $p=0.043$ ) were independent predictive factors for SAP (Table 4). Univariate analysis revealed that ICU admission is related to alcohol, CRP, procalcitonin, and the AIP. In multivariate analysis, the AIP $(\mathrm{OR}=5.099 ; \mathrm{CI}=2.340-11.112 ; p<0.001)$, alcohol, and procalcitonin were independent predictive factors for ICU admission.

Table 4. The association between the AIP and severe acute pancreatitis.

\begin{tabular}{cccccc}
\hline Variable & OR & $p$-Value & OR & 95\% CI & $p$-Value ${ }^{\#}$ \\
\hline Gender (Male) & 0.603 & 0.242 & 0.406 & $0.152-1.084$ & 0.406 \\
Age & 0.995 & 0.688 & 1.006 & $0.975-1.038$ & 0.708 \\
Gallstone & 0.417 & 0.051 & 2.069 & $0.288-14.870$ & 0.470 \\
Alcohol & 2.060 & 0.090 & 5.782 & $1.111-30.088$ & 0.037 \\
Smoking & 1.282 & 0.558 & & & \\
Hypertension & 1.159 & 0.732 & & & \\
Diabetes mellitus & 0.897 & 0.824 & & & \\
Body mass index & 1.067 & 0.157 & 1.077 & $0.975-1.189$ & 0.143 \\
C-reactive protein & 1.031 & 0.214 & & & \\
Procalcitonin & 1.015 & 0.008 & 1.013 & $1.000-1.025$ & 0.043 \\
AIP & 3.119 & $<0.001$ & 4.571 & $1.913-10.922$ & 0.001 \\
\hline
\end{tabular}

* Univariate analysis was performed; ${ }^{\#}$ multivariate analysis was performed; OR: odds ratio; CI: confidence interval; AIP: atherogenic index of plasma. 


\section{Discussion}

In this cross-sectional study, we explored the relationship between the AIP and the severity of $\mathrm{AP}$, and we found that the AIP is higher in patients with severe AP compared to patients with mild or moderately severe AP. We also found that the AIP is positively correlated with the 2012 revised Atlanta classification. Furthermore, the AIP is an independent predictor of SAP, and the predictive ability for SAP is superior to that of either of TG or HDL cholesterol. These results suggest that the AIP may be a simple biomarker for predicting SAP. To our knowledge, this is the first study to evaluate the relationship between the AIP and AP.

Many studies have focused on the early diagnosis and treatment of SAP in order to reduce the high morbidity and mortality of AP. A diagnosis of SAP is based on clinical manifestations, laboratory tests, and imaging. However, it is difficult to predict the course of the disease, as AP is complex and the clinical course varies even when the clinical and radiological scores are the same at the initial diagnosis [18]. Traditional scoring systems are complicated and time consuming, as they are calculated based on several clinical findings and laboratory parameters. Many investigators have tried to find simple and convenient biomarkers to predict the severity of AP. Inflammatory markers, including $\mathrm{CRP}$ and procalcitonin, and various cytokines and chemokines, including tumor necrosis factor- $\alpha$ and interleukin-6, have been evaluated as predictors of SAP, as well as markers of the development of specific OF [19]. Recently, several studies investigated the association between AP and dyslipidemia. Morbid obesity and the presence of metabolic syndrome have shown a higher risk of moderately severe $\mathrm{AP}$ and SAP $[20,21]$. These results suggest that an indicator for both dyslipidemia and inflammatory status could be the ideal biomarker for predicting the severity of AP. The AIP is a comprehensive lipid index and is used as a reasonable marker for both inflammation and impaired lipid metabolism [22,23].

Our study illustrates several important and novel findings. First, there were correlations between an increased AIP and significant increases in the WBC count and serum CRP. As pancreatic inflammation is the most important characteristic feature of AP and involves an excessive recruitment of leukocytes [24], the levels of WBC and CRP have a major role in AP. The WBC count is included in the Ranson score and is one of the clinical criteria of systemic inflammatory response syndrome [6]. Additionally, the relationship between coagulation and inflammation are two-way and are based on positive feedback. Therefore, the development of inflammation leads to the activation of coagulation [25]. The markers of activated coagulation are useful in the early prediction of SAP [26]. Additionally, a previous study has evaluated the predictive value of CRP in AP [27]. Second, the AIP was positively correlated with BMI in our study. Obesity has roles in initiating AP and worsening AP outcomes [28]. Obesity is related with increased gallbladder stones, hypertriglyceridemia, and diabetes. Furthermore, increased intrapancreatic and peripancreatic fat induce local pancreatic necrosis and systemic injury, including OF [29]. Third, the AIP was positively correlated with the 2012 revised Atlanta classification in this study. As this classification system is widely used for the severity stratification of AP, this correlation suggests that a higher AIP might be related to SAP. Finally, the AIP was an independent predictive factor for SAP and ICU admission in this study. This result is in accordance with our hypothesis. As the AIP is easily calculated with the level of TG and HDL, it is a useful biomarker to predict the severity of AP in clinical practice.

The AIP reflects the ratio of serum TG and HDL cholesterol and is calculated as $\log (\mathrm{TG} / \mathrm{HDL})$. Hypertriglyceridemia is a common etiology of AP, and elevated serum TG is independently associated with persistent $\mathrm{OF}[16,30]$. Hypertriglyceridemia-induced pancreatitis is not fully understood, but hypertriglyceridemia is thought to increase plasma viscosity, induce pancreatic ischemia, and trigger organ inflammation [31]. In contrast, decreased HDL cholesterol is related with pancreatic necrosis, persistent OF, and mortality in AP [14,15]. Low HDL, which has anti-inflammatory properties, can lead to a more severe systemic inflammatory response [32]. The AIP has been suggested to be a surrogate of small, dense low-density lipoprotein cholesterol, which plays a central role in the induction of pro-inflammatory mediators and the reduction in inducing anti-oxidants [33]. Therefore, the predictive power of the AIP for SAP may be due to the fact that the AIP reflects the interaction between atherogenic 
and protective lipoprotein. Considering the mechanism of AP pathogenesis, a higher AIP can be a useful biomarker for the prediction of the severity of AP.

There are several limitations to our study. First, the number of SAP cases was small, and the study was conducted at a single institution. However, we enrolled sufficient numbers of all AP severities with different etiologies. Second, as the design of the study was cross-sectional, we cannot determine a cause-and-effect relationship between the AIP and the severity of AP. Finally, inflammatory cytokines such as tumor necrosis factor- $\alpha$ and interleukin- 6 were not measured. However, this study is the first study to demonstrate that the AIP is related to SAP. A large-scale study is needed to establish the clinical role of the AIP for predicting the severity of AP and providing prognoses.

In conclusion, we found that the AIP is significantly associated with SAP and that the AIP is a superior predictive factor to TG or HDL cholesterol level alone for predicting the risk of SAP. Our findings provide robust evidence regarding the association of impaired lipid metabolism and the severity of AP, thereby suggesting that the AIP is a simple and novel biomarker for predicting SAP. As the AIP can be easily measured in a convenient manner, it can be applied in clinical practice.

Author Contributions: Study concept and design, J.H.H., K.J.L.; Acquisition of data, S.K.C., J.W.K.; Interpretation of data and statistical analysis, K.J.L., J.H.H.; Drafting of the manuscript, S.K.C., J.H.H., K.J.L.; Critical revision of the manuscript: J.W.K., J.H.H. All authors have read and agreed to the published version of the manuscript.

Funding: This work was supported by a National Research Foundation of Korea (NRF) grant funded by the Korean government (MSIP) (No. NRF-2019R1F1A1042044 and NRF-2019R1G1A109408).

Acknowledgments: We thank to Geun Sook Lee for coordinating this study.

Conflicts of Interest: No conflict of interest relevant to this article was reported.

\section{References}

1. Yadav, D.; Lowenfels, A.B. Trends in the epidemiology of the first attack of acute pancreatitis: A systematic review. Pancreas 2006, 33, 323-330. [CrossRef]

2. Crockett, S.D.; Wani, S.; Gardner, T.B.; Falck-Ytter, Y.; Barkun, A.N.; American Gastroenterological Association Institute Clinical Guidelines, Committee. American gastroenterological association institute guideline on initial management of acute pancreatitis. Gastroenterology 2018, 154, 1096-1101. [CrossRef] [PubMed]

3. Koutroumpakis, E.; Slivka, A.; Furlan, A.; Dasyam, A.K.; Dudekula, A.; Greer, J.B.; Whitcomb, D.C.; Yadav, D.; Papachristou, G.I. Management and outcomes of acute pancreatitis patients over the last decade: A us tertiary-center experience. Pancreatology 2017, 17, 32-40. [CrossRef] [PubMed]

4. $\quad$ Banks, P.A.; Bollen, T.L.; Dervenis, C.; Gooszen, H.G.; Johnson, C.D.; Sarr, M.G.; Tsiotos, G.G.; Vege, S.S.; Acute Pancreatitis Classification Working, G. Classification of acute pancreatitis-2012: Revision of the atlanta classification and definitions by international consensus. Gut 2013, 62, 102-111. [CrossRef] [PubMed]

5. Van Dijk, S.M.; Hallensleben, N.D.L.; van Santvoort, H.C.; Fockens, P.; van Goor, H.; Bruno, M.J.; Besselink, M.G.; Dutch Pancreatitis Study, G. Acute pancreatitis: Recent advances through randomised trials. Gut 2017, 66, 2024-2032. [CrossRef] [PubMed]

6. Ranson, J.H.; Rifkind, K.M.; Roses, D.F.; Fink, S.D.; Eng, K.; Localio, S.A. Objective early identification of severe acute pancreatitis. Am. J. Gastroenterol. 1974, 61, 443-451.

7. Larvin, M.; McMahon, M.J. Apache-ii score for assessment and monitoring of acute pancreatitis. Lancet 1989, 2, 201-205. [CrossRef]

8. Balthazar, E.J.; Robinson, D.L.; Megibow, A.J.; Ranson, J.H. Acute pancreatitis: Value of CT in establishing prognosis. Radiology 1990, 174, 331-336. [CrossRef] [PubMed]

9. Wu, B.U.; Johannes, R.S.; Sun, X.; Tabak, Y.; Conwell, D.L.; Banks, P.A. The early prediction of mortality in acute pancreatitis: A large population-based study. Gut 2008, 57, 1698-1703. [CrossRef]

10. Riche, F.C.; Cholley, B.P.; Laisne, M.J.; Vicaut, E.; Panis, Y.H.; Lajeunie, E.J.; Boudiaf, M.; Valleur, P.D. Inflammatory cytokines, $\mathrm{c}$ reactive protein, and procalcitonin as early predictors of necrosis infection in acute necrotizing pancreatitis. Surgery 2003, 133, 257-262. [CrossRef]

11. Zhang, X.; Zhang, X.; Li, X.; Feng, J.; Chen, X. Association of metabolic syndrome with atherogenic index of plasma in an urban chinese population: A 15-year prospective study. Nutr. Metab. Cardiovasc. Dis. 2019, 29, 1214-1219. [CrossRef] 
12. Cai, G.; Liu, W.; Lv, S.; Wang, X.; Guo, Y.; Yan, Z.; Du, Y.; Zhou, Y. Gender-specific associations between atherogenic index of plasma and the presence and severity of acute coronary syndrome in very young adults: A hospital-based observational study. Lipids Health Dis. 2019, 18, 99. [CrossRef] [PubMed]

13. Navina, S.; Acharya, C.; DeLany, J.P.; Orlichenko, L.S.; Baty, C.J.; Shiva, S.S.; Durgampudi, C.; Karlsson, J.M.; Lee, K.; Bae, K.T.; et al. Lipotoxicity causes multisystem organ failure and exacerbates acute pancreatitis in obesity. Sci. Transl. Med. 2011, 3, 107ra110. [CrossRef] [PubMed]

14. Khan, J.; Nordback, I.; Sand, J. Serum lipid levels are associated with the severity of acute pancreatitis. Digestion 2013, 87, 223-228. [CrossRef] [PubMed]

15. Zhang, Y.; Guo, F.; Li, S.; Wang, F.; Meng, Z.; Zhao, J.; Liu, Z.; Wang, B.; Fan, P.; Wang, C.; et al. Decreased high density lipoprotein cholesterol is an independent predictor for persistent organ failure, pancreatic necrosis and mortality in acute pancreatitis. Sci. Rep. 2017, 7, 8064. [CrossRef] [PubMed]

16. Nawaz, H.; Koutroumpakis, E.; Easler, J.; Slivka, A.; Whitcomb, D.C.; Singh, V.P.; Yadav, D.; Papachristou, G.I. Elevated serum triglycerides are independently associated with persistent organ failure in acute pancreatitis. Am. J. Gastroenterol. 2015, 110, 1497-1503. [CrossRef]

17. Working Group IAP/APA Acute Pancreatitis Guidelines. IAP/APA evidence-based guidelines for the management of acute pancreatitis. Pancreatology 2013, 13, e1-e15. [CrossRef] [PubMed]

18. Zerem, E. Treatment of severe acute pancreatitis and its complications. World J. Gastroenterol. 2014, 20, 13879-13892. [CrossRef]

19. Papachristou, G.I. Prediction of severe acute pancreatitis: Current knowledge and novel insights. World J. Gastroenterol. 2008, 14, 6273-6275. [CrossRef]

20. Krishna, S.G.; Hinton, A.; Oza, V.; Hart, P.A.; Swei, E.; El-Dika, S.; Stanich, P.P.; Hussan, H.; Zhang, C.; Conwell, D.L. Morbid obesity is associated with adverse clinical outcomes in acute pancreatitis: A propensity-matched study. Am. J. Gastroenterol. 2015, 110, 1608-1619. [CrossRef]

21. Mikolasevic, I.; Milic, S.; Orlic, L.; Poropat, G.; Jakopcic, I.; Franjic, N.; Klanac, A.; Kristo, N.; Stimac, D. Metabolic syndrome and acute pancreatitis. Eur. J. Intern. Med. 2016, 32, 79-83. [CrossRef] [PubMed]

22. Mazidi, M.; Katsiki, N.; Mikhailidis, D.P.; Banach, M. Association of ideal cardiovascular health metrics with serum uric acid, inflammation and atherogenic index of plasma: A population-based survey. Atherosclerosis 2019, 284, 44-49. [CrossRef] [PubMed]

23. Zhu, X.; Yu, L.; Zhou, H.; Ma, Q.; Zhou, X.; Lei, T.; Hu, J.; Xu, W.; Yi, N.; Lei, S. Atherogenic index of plasma is a novel and better biomarker associated with obesity: A population-based cross-sectional study in china. Lipids Health Dis. 2018, 17, 37. [CrossRef] [PubMed]

24. Bhatia, M.; Wong, F.L.; Cao, Y.; Lau, H.Y.; Huang, J.; Puneet, P.; Chevali, L. Pathophysiology of acute pancreatitis. Pancreatology 2005, 5, 132-144. [CrossRef] [PubMed]

25. Danckwardt, S.; Hentze, M.W.; Kulozik, A.E. Pathologies at the nexus of blood coagulation and inflammation: Thrombin in hemostasis, cancer, and beyond. J. Mol. Med. 2013, 91, 1257-1271. [CrossRef]

26. Dumnicka, P.; Maduzia, D.; Ceranowicz, P.; Olszanecki, R.; Drozdz, R.; Kusnierz-Cabala, B. The interplay between inflammation, coagulation and endothelial injury in the early phase of acute pancreatitis: Clinical implications. Int. J. Mol. Sci. 2017, 18, 354. [CrossRef] [PubMed]

27. Stirling, A.D.; Moran, N.R.; Kelly, M.E.; Ridgway, P.F.; Conlon, K.C. The predictive value of c-reactive protein (crp) in acute pancreatitis-is interval change in crp an additional indicator of severity? HPB 2017, 19, 874-880. [CrossRef]

28. Khatua, B.; El-Kurdi, B.; Singh, V.P. Obesity and pancreatitis. Curr. Opin. Gastroenterol. 2017, 33, $374-382$. [CrossRef] [PubMed]

29. Bakker, O.J.; van Santvoort, H.; Besselink, M.G.; Boermeester, M.A.; van Eijck, C.; Dejong, K.; van Goor, H.; Hofker, S.; Ahmed Ali, U.; Gooszen, H.G.; et al. Extrapancreatic necrosis without pancreatic parenchymal necrosis: A separate entity in necrotising pancreatitis? Gut 2013, 62, 1475-1480. [CrossRef] [PubMed]

30. Mosztbacher, D.; Hanak, L.; Farkas, N.; Szentesi, A.; Miko, A.; Bajor, J.; Sarlos, P.; Czimmer, J.; Vincze, R.; Hegyi, P.J.; et al. Hypertriglyceridemia-induced acute pancreatitis: A prospective, multicenter, international cohort analysis of 716 acute pancreatitis cases. Pancreatology 2020, 20, 606-608. [CrossRef]

31. De Pretis, N.; Amodio, A.; Frulloni, L. Hypertriglyceridemic pancreatitis: Epidemiology, pathophysiology and clinical management. United Eur. Gastroenterol. J. 2018, 6, 649-655. [CrossRef] [PubMed] 
32. Murphy, A.J.; Woollard, K.J. High-density lipoprotein: A potent inhibitor of inflammation. Clin. Exp. Pharmacol. Physiol. 2010, 37, 710-718. [CrossRef] [PubMed]

33. Dobiasova, M.; Frohlich, J. The plasma parameter $\log (\mathrm{tg} / \mathrm{hdl}-\mathrm{c})$ as an atherogenic index: Correlation with lipoprotein particle size and esterification rate in apob-lipoprotein-depleted plasma (fer(hdl)). Clin. Biochem. 2001, 34, 583-588. [CrossRef] 